\title{
ARFIMA-GARCH modeling of HRV: clinical application in Acute Brain Injury*
}

\author{
Rute Almeida ${ }^{\dagger}$ \\ rbalmeidefc.up.pt
}

\author{
Celeste Dias $\ddagger$ \\ mcdias@med.up.pt
}

Ana Paula Rocha

aprocha@fc.up.pt

March 2017

\author{
Maria Eduarda Silva ${ }^{\S}$ \\ mesilva@fep.up.pt
}

\begin{abstract}
In the last decade, several HRV based novel methodologies for describing and assessing heart rate dynamics have been proposed in the literature with the aim of risk assessment. Such methodologies attempt to describe the non-linear and complex characteristics of HRV, and hereby the focus is in two of these characteristics, namely long memory and heteroscedasticity with variance clustering. The ARFIMA-GARCH modeling considered here allows the quantification of long range correlations and time-varying volatility. ARFIMA-GARCH HRV analysis is integrated with multimodal brain monitoring in several acute cerebral phenomena such as intracranial hypertension, decompressive craniectomy and brain death. The results indicate that ARFIMAGARCH modeling appears to reflect changes in Heart Rate Variability (HRV) dynamics related both with the Acute Brain Injury (ABI) and the medical treatments effects.
\end{abstract}

\section{INTRODUCTION}

The combination of sympathetic nervous system and parasympathetic nervous system activation defined as Autonomic Nervous System (ANS) tonus, is a function of both central mechanisms and feedback from peripheral systems that might be impaired in critically ill patients with Acute Brain Injury (ABI) [15, 9]. The incidence of ANS dysfunction after ABI is not exactly known, but changes are thought to be related to the severity of injury, to clinical management and, more importantly, seem to correlate independently with increased morbidity and mortality [55, 6, 34]. Heart rate variability (HRV) is currently defined by the fluctuating time between normal sinus beats (RR intervals) [51], and indicates modulation of the heart rate by the ANS [46], baroreceptor function, thermoregulation, vasomotor tone and circadian rhythms [38]. HRV measurements are easy to perform, non-invasive, and have good reproducibility, if used under standardized conditions such as those set by the Task Force of the European Society of Cardiology and the North American Society of Pacing and Electrophysiology in 1996 [49].

\footnotetext{
*Accepted author's manuscript (AAM) to be published in [Complexity and Nonlinearity in Cardiovascular Signals, Springer 2017]. [DOI:10.1007/978-3-319-58709-7_ 17]

${ }^{\dagger}$ CMUP and BSICoS, Universidade do Porto, Porto, Portugal

${ }^{\ddagger}$ CHSJ and FMUP, Universidade do Porto, Porto, Portugal

${ }^{\S}$ CIDMA and FEP, Universidade do Porto, Porto, Portugal

${ }^{\top}$ CMUP and FCUP, Universidade do Porto, Porto, Portugal
} 
Traditional HRV techniques are mostly based in the analysis of RR intervals either in time or frequency domains. The resulting indexes include standard deviation measures, power spectral measures in clinically relevant frequency bands, and ratio between low and high frequency activity (ANS branches balance). Since 1965 when Vallbona et al [50] linked HRV with the severity of brain injury and brain death risk, several other authors reported correlations between HRV, neuromonitoring variables and outcome after ABI [55, 6, 15, 13, 34, 33, 21, 47]. However, traditional approaches are unable to deal with high non stationarity of long HRV segments, specially marked in critically ill patients. Therefore early studies were restricted to short intervals limiting the development of dynamic prognostic tools in ABI [19].

In the last decades several new methodologies to analyse HRV have been proposed in the literature accompanied by their application in different physiological and clinical studies [38, 21, 44]. These methods comprehend, by a broad definition in [44], estimation of long-range correlation and fractal scaling, quantifying short-term complexity, entropy and regularity measures, analysis of chaotic behaviour with nonlinear dynamical systems. Alternatively, several authors proposed model based approaches to HRV analysis which cope with the nonstationarity of the data. One approach is time-variant AutoRegressive (AR) analysis using exponentially smoothed recursive least squares estimation, with fixed and varying forgetting factors, [5, 41, 31]. Another approach is based on the adaptive segmentation of the non stationary record into approximately stationary records [36] which are usually modeled with short memory AR models. This procedure leads to linear parametric models for the conditional mean which, however, do not capture the long range correlations of HRV data. To overcome this drawback, Leite and co-authors [22] proposed the use of Fractionally Integrated AutoRegressive Moving Average (ARFIMA) models, an extension of the well-known AutoRegressive Moving Average (ARMA) models, to represent both short and long term behaviours of HRV series. The authors use adaptive segmentation of 24-hour recordings of HRV to find that the long memory parameter changes with time and between day and night periods. These findings were later corroborated by Baillie et al [3].

ARFIMA models describe the conditional mean of the data, however HRV exhibits also changes in variance over time, with periods of large variability followed by periods of stability, suggesting heteroscedasticity (varying variance). These volatility clustering phenomena may be well described by conditional volatility models such as the Generalized AutoRegressive Conditionally Heteroscedastic (GARCH), [8]. Consequently, Leite and co-authors [24, 25] considered the joint modeling of long-memory and heteroscedasticity characteristics of HRV using fractionally integrated ARFIMA models with GARCH innovations. In these works ARFIMA-GARCH modeling is used to capture and remove long-range correlation and estimate conditional volatility in 24-hour HRV recordings from Noltisalis database [45] and the database Is the Normal Heart Rate Chaotic? 1] from Physionet [14], respectively, allowing to discriminate between health and disease. A further empiric characteristic of HRV volatility is asymmetry in response to shocks. Leite and co-authors [27, 42] used exponential GARCH (ARFIMA-EGARCH) models to capture these effects and found that the parameters of the models are promising in differentiating health and disease. Note that GARCH models were first applied in the HRV context to develop an HRV based apnea screening tool [17], with the mean of the data described by a simple AR(1) process.

Rocha and co-authors [40] applied the ARFIMA-GARCH approach to the analysis of pediatric patients with acute brain injury. In this study, the parameters for long-memory and conditional variance estimated from cases with posterior confirmation of brain death (BD) differ significantly from those estimated from survivors and seem able to contribute to characterize disease severity in children with acute brain injury.

In spite of the dynamics of the ABI and the complexity of the Neurocritical Care Unit (NCCU) management, HRV analysis is a valuable tool to investigate the sympathetic and parasympathetic dysfunction of the ANS after ABI [47]. HRV has not been widely adopted by neurointensivists, nevertheless its enormous potential for application at bedside after ABI.

In this chapter are considered some clinical conditions that frequently occur in the NCCU

\footnotetext{
${ }^{1}$ https://www.physionet.org/challenge/chaos/
} 
where ARFIMA-GARCH HRV analysis integrated with multimodal brain monitoring may help clinicians to interpret or predict, in a more comprehensible way, acute cerebral phenomena such as intracranial hypertension with: plateau waves, decompressive craniectomy and brain death.

\section{ARFIMA-GARCH models}

This section describes a class of models appropriate to characterize the persistence (long memory) and nonlinear characteristics of RR series.

The characteristic of persistence, long-memory or long range dependence appears in many natural phenomena and is characterized by slowly decaying serial correlations, or equivalently by a spectrum with an hyperbolic behaviour at the origin, $f(\lambda) \sim|\lambda|^{-2 d}$. Long memory is thus related to self-similar processes also known as fractals and the so called $1 / f$ noise. Heuristic methods, such as the Detrended Fluctuation Analysis (DFA), the Rescaled Range (R/S) and Rescaled Variance (R/V) are often used for assessing long memory in data but are mainly useful for descriptive purposes rather than concrete statistical inference or model building. The alternative taken herein is to consider a model based approach with ARFIMA models (sometimes also designated by FARIMA). These models extend the well known ARMA models for time series and are, thus, able to model the short as well as the long memory of the data.

GARCH models were introduced in the literature to account for the following nonlinear behaviour of many data sets: heteroscedasticity in the conditional variance with clusters of volatility. This same phenomena has been observed in RR series [24, 25]. To combine the persistence in mean with conditional time-varying variance in a model based approach, ARFIMA models with GARCH innovations are considered. The ARFIMA-GARCH models is a wide classe of models which is able to cater for complex nonlinearities in dynamics of the data.

$\operatorname{ARFIMA}(p, d, 0)-\operatorname{GARCH}(1,1)$ models are defined as follows

$$
\begin{gathered}
\phi(B)(1-B)^{d} x_{t}=\varepsilon_{t}, \\
\varepsilon_{t}=\sigma_{t} z_{t}, \\
\sigma_{t}^{2}=\operatorname{Var}\left(\varepsilon_{t} \mid \mathscr{F}_{t-1}\right)=u_{0}+v_{1} \sigma_{t-1}^{2}+u_{1} \varepsilon_{t-1}^{2}
\end{gathered}
$$

where $B$ is the backward-shift operator, $d \in \mathbb{R}$ is a real number, $(1-B)^{d}=\sum_{k=0}^{\infty}\left(\begin{array}{c}d \\ k\end{array}\right)(-1)^{k} B^{k}$ is the fractional difference operator, [4], $\phi(B)=1-\phi_{1} B-\ldots-\phi_{p} B^{p}$ is a polynomial in $B, u_{0}>0$, $u_{1}, v_{1} \geq 0, p \in \mathbb{N}_{0}, z_{t}$ are independent and identically distributed random variables with zero mean and unit variance and $\mathscr{F}_{t}$ denotes the history of the process up to time $t$. Equation (1) describes the conditional mean of the process with serially uncorrelated residuals or shocks and is called an $\operatorname{ARFIMA}(p, d, 0)$ model [4]. Equations (2) and (3) describe the conditional variance of the process and define a GARCH $(1,1)$ model $[8]$.

In equation (1) parameter $d$ determines the long-term behaviour in mean 2 , whereas $p$ and the coefficients in $\phi(B)$ allow for the modeling of short-range properties. The model is stationary for $-0.5<d<0.5$, nonstationary but mean reverting for $0.5 \leq d<1$. Equation (3) describes the dynamics of the conditional variance (volatility) of the process: $\sigma_{t}^{2}$ is dependent on its own lagged value and on the past squared shocks or residuals from the mean equation. Parameter $u_{1}$ characterizes the short-range properties in volatility and parameter $v_{1}$ characterizes persistence in the volatility. The $\operatorname{GARCH}(1,1)$ model is second order stationary if $v_{1}+u_{1}<1$, [8]. Therefore the $\operatorname{ARFIMA}(p, d, 0)$-GARCH $(1,1)$ is stationary if $-0.5<d<0.5$, all the roots of $\phi(B)$ lie outside the unit circle and $v_{1}+u_{1}<1$.

The spectral density function of a stationary $\operatorname{ARFIMA}(p, d, 0)$ process is given by [16]

$$
f_{\omega}=f_{\omega}^{*}\left|1-e^{-i \omega}\right|^{-2 d}, \quad-\pi \leq \omega \leq \pi,
$$

\footnotetext{
${ }^{2}$ The long memory parameter is related to the Hurst coefficient, $H=d+0.5$, to the fractal dimension, $D=2-H$ and to the slope of the (generalized) spectral density in the low frequency range by $\alpha=2 d$.
} 


$$
f_{\omega}^{*}=\frac{\sigma_{\varepsilon}^{2}}{\left|\phi\left(e^{-i \omega}\right)\right|^{2}}
$$

where

$$
\sigma_{\varepsilon}^{2}=\operatorname{var}\left(\varepsilon_{t}\right)=\frac{u_{0}}{1-v_{1}-u_{1}}
$$

and $f_{\omega}^{*}$ is the spectral density of the $\operatorname{AR}(p)$ process. Since the autocorrelation function of a $\operatorname{GARCH}(1,1)$ process is the same as that of white noise, [8], the spectral density function of a stationary $\operatorname{ARFIMA}(p, d, 0)$ - $\operatorname{GARCH}(1,1)$ process is given by equations $(4)$ and $(5)$ with $\sigma_{\varepsilon}^{2}$ defined by (6). For $0.5 \leq d<1$ equation (4) corresponds to a pseudo-spectral density [18, 52], since the process is nonstationary but mean reverting.

A final note regarding ARFIMA $(p, d, 0)$ models is due. The model may be extended to nonstationary settings in which $d=d_{L M}+D>1$ with $-0.5<d_{L M}<0.5$ and $D \in\{1,2, \ldots\}$. The most usual case occurs with $D=1$, when the process is said to have a unit root: the $\operatorname{ARFIMA}(p, d, 0)$ is, then, used to model the increments of the series, that is the differences between consecutive observations.

Given a time series $x_{1}, \ldots, x_{n}$, to estimate the parameters of an $\operatorname{ARFIMA}(p, d, 0)-\operatorname{GARCH}(1,1)$, model proceed as follows [25]:

(i) estimate $-0.5<d<0.5$ using the semi-parametric local Whittle estimator [39];

(ii) define the filtered data $y_{t}=(1-B)^{d} x_{t}$;

(iii) estimate the $\operatorname{AR}(p)-\operatorname{GARCH}(1,1)$ parameters in the filtered data $y_{t}$ by maximum likelihood.

The semi-parametric local Whittle estimator of the long memory, step $(i)$, is an estimator in the Fourier domain, and acknowledged in the literature for its statistical properties, efficiency and asymptotic normality, and also easiness of implementation, [4]. Other estimators, such as the logwavelet regression estimator which is a semiparametric estimator in the wavelet domain, have the disadvantage of complicated asymptotic distributions. It is worth noting that the standard error of the estimator in $(i)$ is quite large, $\sqrt{1 /\left(4 n^{0.8}\right)}$ : for example, the standard error associated with the estimate of $d$ from a data set with $n=2000$ observations is 0.024 . Under the ARFIMA model based approach it is also necessary determine the order $p$ of the AR component of the model. This is accomplished in step (iii) using Akaike Information Criterion, AIC, under maximum likelihood estimation. The conditional heteroscedasticity in the series, that is the need for the GARCH component, is assessed by the Ljung-Box test in the squared residuals, [28]. If the series does not present heteroscedasticity then the final model is an $\operatorname{ARFIMA}(p, d, 0)$. In any case, only models with uncorrelated residuals, checked by the Ljung-Box test, are considered valid models.

To illustrate the use of ARFIMA $(p, d, 0)$-GARCH $(1,1)$ models in HRV data consider Figures 1 to 3. each one representing the analysis of a segment of the tachogram of a patient in intensive care. Figure 1 represents in (a) the tachogram of a segment with 1571 beats and in (b) the corresponding sample autocorrelation function (SACF) in black and the ACF of the best AR model for the data in gray. The SACF shows a very slow decay indicating that the dependence between distant observations is not negligible and thus displaying long memory characteristics. $\operatorname{An} \operatorname{ARFIMA}(p=26, d=0.19,0)$ model is fitted to this segment. The resulting residuals $\left(\varepsilon_{t}\right)$, displayed in (c) exhibit little correlation, (d), indicating that the ARFIMA model is adequate to explain the dynamics and conditional mean of the data. Also, the squared residuals in (e) exhibit no significant autocorrelation in (f), indicating absence of time-varying conditional variance or heteroscedasticity. These results are confirmed by the $p$-value $<0.001$ of Ljung-Box test applied to the squared residuals of ARFIMA. A similar analysis is depicted in Figure 2 but it is worth noting that the SACF in (b) indicates a much stronger long-memory effect which is confirmed by the value $d=0.61$ estimated from the data. The last illustration is depicted in Figures 3 . Once again the SACF in (b) of the tachogram segment in (a) indicates the existence of long memory. The residuals $\varepsilon_{t}$ displayed in (c) and the corresponding SACF in (d), exhibit little correlation indicating that the estimated $\operatorname{ARFIMA}(p=19, d=0.82,0)$ model is adequate to explain the dynamics and conditional mean of the data. However, the squared residuals in (e) exhibit significant 
autocorrelation in (f), indicating time-varying conditional variance or heteroscedasticity. These results are confirmed by the $p$-value $>0.05$ of Ljung-Box test applied to the squared residuals of ARFIMA. Now, to model this effect a $\operatorname{GARCH}(1,1)$ model is entertained for the ARFIMA residuals. The estimates for the parameters are $u_{1}=0.05, v_{1}=0.94$. The squared residuals from the ARFIMA-GARCH $(1,1)$ model and corresponding SACF, $(\mathrm{g})$ and $(\mathrm{h})$, indicate absence of heteroscedasticity. Finally, the volatility of this HRV segment is depicted in (i).

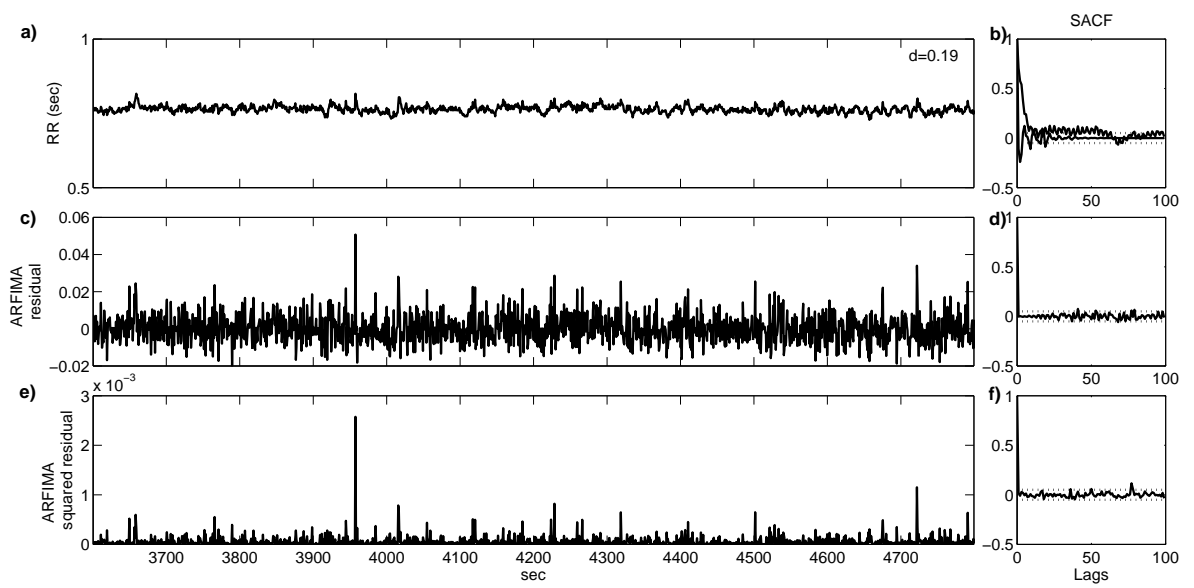

Figure 1: (a) Tachogram (20 min segment with 1571 beats of RR series) with $d=0.19$, (b) SACF (black) and AR ACF (grey), (c) and (d) ARFIMA residuals and corresponding SACF, (e) and (f) squared residuals and SACF. The horizontal lines (- -) show the $95 \%$ confidence limits for SACF.

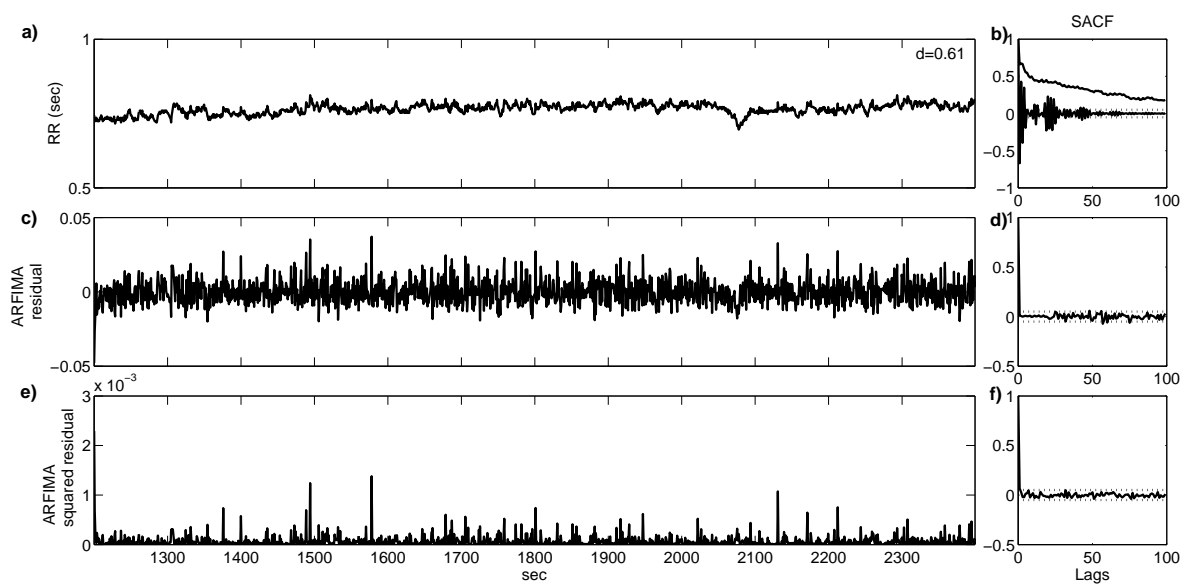

Figure 2: (a) Tachogram (20 min segment with 1569 beats of RR series) with $d=0.61$, (b) SACF (black) and AR ACF (grey), (c) and (d) ARFIMA residuals and corresponding SACF, (e) and (f) squared residuals and SACF. The horizontal lines (- -) show the $95 \%$ confidence limits for SACF.

ARFIMA-GARCH models provide a set of parameters $\left(\phi_{1}, \ldots, \phi_{p}, p, d, u, v\right)$ that can be used to describe linear and non-linear characteristics of HRV. The ARFIMA parameter $d$ determines the long-term behaviour in mean, while GARCH parameters $u$ and $v$ describe the non-linear behaviour of conditional time-variant variance. The order $p$ and the coefficients $\phi_{1}, \ldots, \phi_{p}$ of the AR component of the ARFIMA-GARCH model describe the short memory characteristics, as the usual AR aproach to HRV modeling. Consequently as the ARFIMA-GARCH spectrum is 

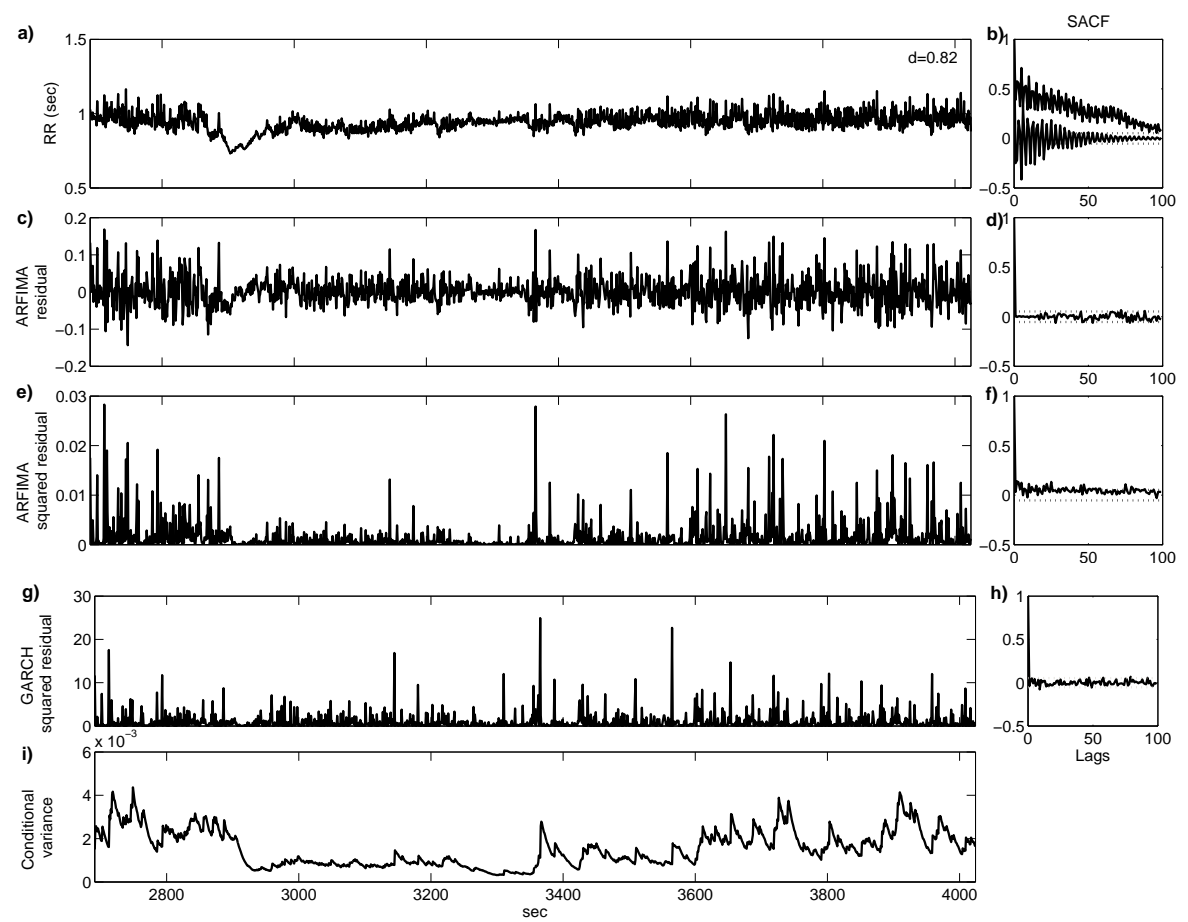

Figure 3: (a) Tachogram (22 min segment with 1420 beats of RR series) with $d=0.82$, (b) SACF (black) and AR ACF (grey), (c) and (d) ARFIMA residuals and corresponding SACF, (e) and (f) squared residuals and SACF, (g) and (h) ARFIMA-GARCH residuals and SACF, (i) conditional volatility. The horizontal lines (- -) show the $95 \%$ confidence limits for SACF.

the same as that of ARFIMA, the spectral indexes extracted from the ARFIMA-GARCH model provide the same information on HRV as that provided by AR models. The advantage is that the estimation of $d$ allows an individualized data based removal of the very low frequency component and thus avoiding any additional filtering.

ARFIMA-GARCH models are applied in Section 4 to data from adult traumatic brain injury patients described in the next section.

\section{Data, pre-processing and HRV modeling}

The data analysed in the remainder of this chapter regard a retrospective study of five adult severe traumatic brain injury patients (TBI), admitted to a NCCU who developed intracranial hypertension. The exclusion criteria were pregnancy and age ( $\leq 18$ years). Standard management of patients included sedation and analgesia with propofol and/or midazolam and fentanyl and normoventilation. Intracranial Pressure (ICP) and Cerebral Perfusion Pressure (CPP) management was achieved according to the NCCU protocol previously published [11], and approved by the hospital Ethics Committee. The physiological variables continuously monitored included ECG at $250 \mathrm{~Hz}$, heart rate (HR), arterial blood pressure (ABP), ICP, amplitude of ICP (AMP), CPP, Endtidal $\mathrm{CO} 2$ (ETCO2), brain tissue oxygenation (pbtO2), cerebral blood flow (CBF) and cerebral autoregulation (CAR) evaluated with cerebrovascular pressure reactivity index (PRx). Software $\mathrm{ICM}+\mathrm{R}$ was used to collect primary data and continuously record raw signals that were analysed offline. Automatic ECG annotation was performed with a multi-scale wavelet-based ECG annotator previously developed and validated [32] using MATLAB ${ }^{\circledR}$ and BioSigBrowser [7] tools. The RR series were defined as the time interval, in seconds, between consecutive QRS automatic 
locations; RR values admitted have a maximum of $3 \mathrm{sec}$ and are within a 3/0.6745 tolerance on the difference to Median Absolute Deviation, [54]. The series were divided into segments with minimum 1024 and maximum 2048 consecutive beats and assumed to be sampled at the local mean heart rate.

ARFIMA-GARCH modeling of each RR series segment, $x_{t}$, described in Section 2 by steps (i),(ii) and (iii) assumes that the segments $x_{t}$ are approximately stationary. However, since this is not always the case, additional steps must be performed to ensure that the model are estimated over stationary segments. First, the segment is tested for stationarity using the Augmented-DickeyFuller test for unit roots, [30]. If there is a unit root, then $D=1$, c.f. section 2 and the RR series is differenced. This means that step $(i)$ is performed over the differenced RR or, in other words, the series of RR increments, $\Delta \mathrm{RR}=x_{t}-x_{t-1}$. The resulting estimate $d_{L M}^{\Delta \mathrm{RR}}$ is expected to belong to the interval ] $-0.5,0.5$ [ otherwise over-differencing may have occurred. However, in view of the large standard error associated to the estimates for $d$, see section 2, a shorter, more robust interval is considered for this purpose. Thus, if $d_{L M}^{\Delta \mathrm{RR}}>-0.45$ then set $d=1+d_{L M}^{\Delta \mathrm{RR}}$ in step (ii), and proceed to step (iii). Otherwise, if $d_{L M}^{\Delta \mathrm{RR}}<-0.45$ steps $(i), \quad$ (ii), (iii) are performed over the RR series $x_{t}$. Also, if there is no unit root, steps $(i),(i i)$, (iii) are performed over the RR series $x_{t}$. The order of the $\operatorname{AR}(p)$ model is step (iii) is selected in the range 6 to 36 according AIC. An RR segment is said to have a valid ARFIMA model if the residuals of the ARFIMA model are uncorrelated, tested by Ljung-Box at 5\% level of significance. Similarly, an RR segment is said to have a valid ARFIMA-GARCH(1,1) model if the residuals of the ARFIMA model are uncorrelated but its squared residuals are correlated, all tested by Ljung-Box at $5 \%$ level of significance.

Finally and for comparison purposes, the RR segments are firstly detrended using smooth priors $(\lambda=50),[48]$ and then modeled by an AR model with adequate order chosen by AIC.

For each fitted model, parametric HRV measures were obtained using spectral AR decomposition [1] by assigning each pole contribution to the spectral band in which the pole is located according to the standard fixed bands defined in [49]: low frequency (LF: 0.04-0.15 Hz) and high frequency $(0.15-1.4 \mathrm{~Hz}$ ) bands, all frequencies in spectra (TP). LFn and HFn are measures, respectively at LF and HF bands, normalized by power in the band above $0.04 \mathrm{~Hz}$. Autonomic balance B was obtained as LF/HF.

\section{Clinical applications in Acute Brain Injury}

ANS dysfunction in ABI leads to changes in HRV which appear to be particularly marked in patients subsequently declared with Brain Death (BD). It is well known that reduced HRV, assessed in time, frequency and complexity, is a risk index after trauma for both morbidity and mortality and an early predictor of BD. Previous studies on cardiovascular series variability indicate that the derived indexes may provide an early complementary tool for time course prediction and prognostic in critical illness [2, 43]. Although some authors have proposed HRV as an auxiliary tool in trauma, it is not usually used in the clinical practice for ABI patients, nor considered as a decision support tool for the commencement of the proceeding for BD declaration [33, 43, 35]. This work aims at illustrating the potential of ARFIMA-GARCH modeling of HRV, integrated with multimodal brain monitoring, for helping clinicians to interpret or predict in a more comprehensible way acute cerebral phenomena such as intracranial hypertension with: a) plateau waves, b) decompressive craniectomy, c) brain death.

Plateau waves (PW) are sudden rises in ICP, up to 40-100 $\mathrm{mmHg}$, lasting more than 5 minutes, terminating either spontaneously or in response to active treatment [10, 29]. Meanwhile arterial blood pressure remains stable or varies modestly, as illustrated in Figure 4. Decompressive craniectomy (DC) is a surgical option for managing refractory intracranial hypertension that remains controversial. DC is efficacious in reducing ICP, as illustrated in Figure 5, by the removal of a bone flap allowing brain volume expansion. The focus of current debate surrounds identification of the subset of patients for whom it is appropriate to offer DC [20]. Intracranial 
hypertension may also evolve to brain death (BD) due to the absence of blood flow, zero flow $(\mathrm{ZF})$, in consequence of cerebral circulatory arrest leading to the complete and irreversible loss of brain stem function [53] as illustrated in Figure 6.

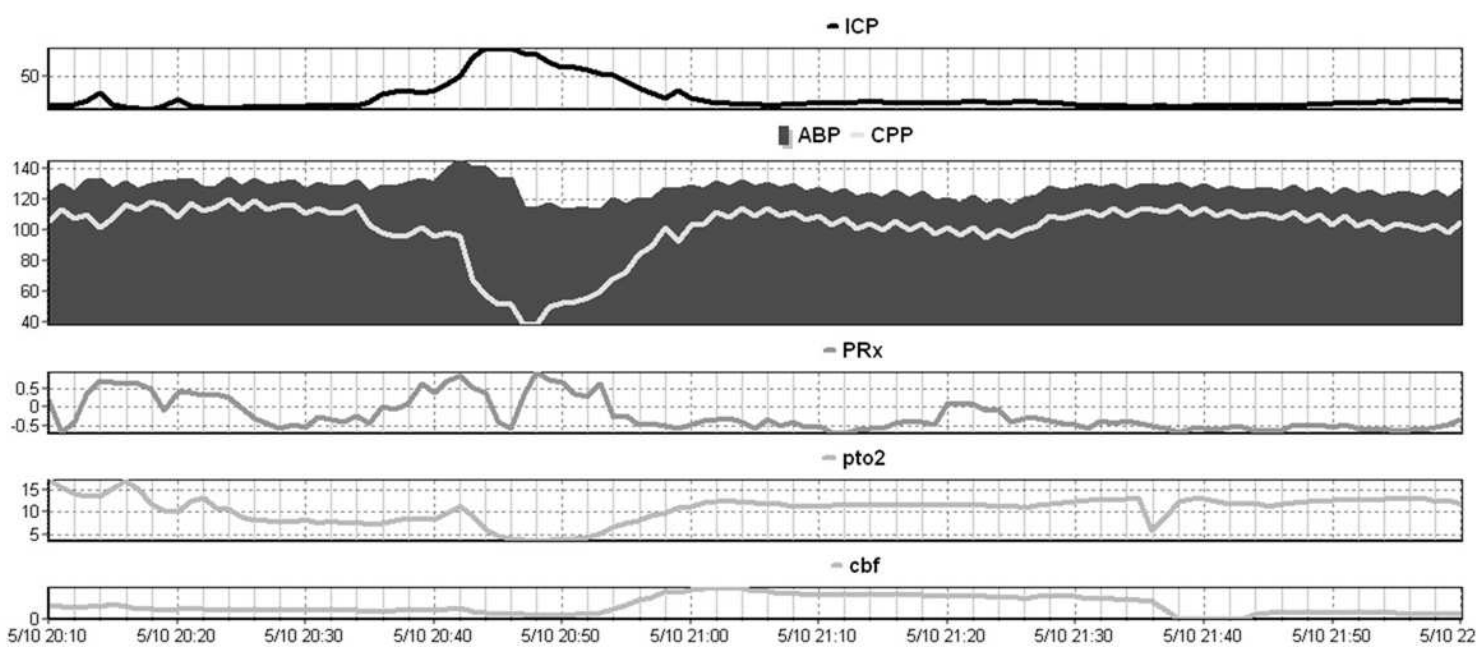

Figure 4: Plateau wave (PW) documentation with multimodal brain monitoring using intracranial pressure (ICP), mean arterial blood pressure (ABP), cerebral perfusion pressure (CPP), brain tissue oxygenation pressure (pbtO2), cerebral blood flow $(\mathrm{CBF})$ and cerebral autoregulation (CAR) evaluation with pressure reactivity index (PRx). During PW while ICP increases, CPP, pbtO2 and CBF decreases along with impairment of CAR (PRx $>0.3$ ). After PW, CPP and PRx recover but pbtO2 and CBF increase because of hyperaemic response after ischemia.

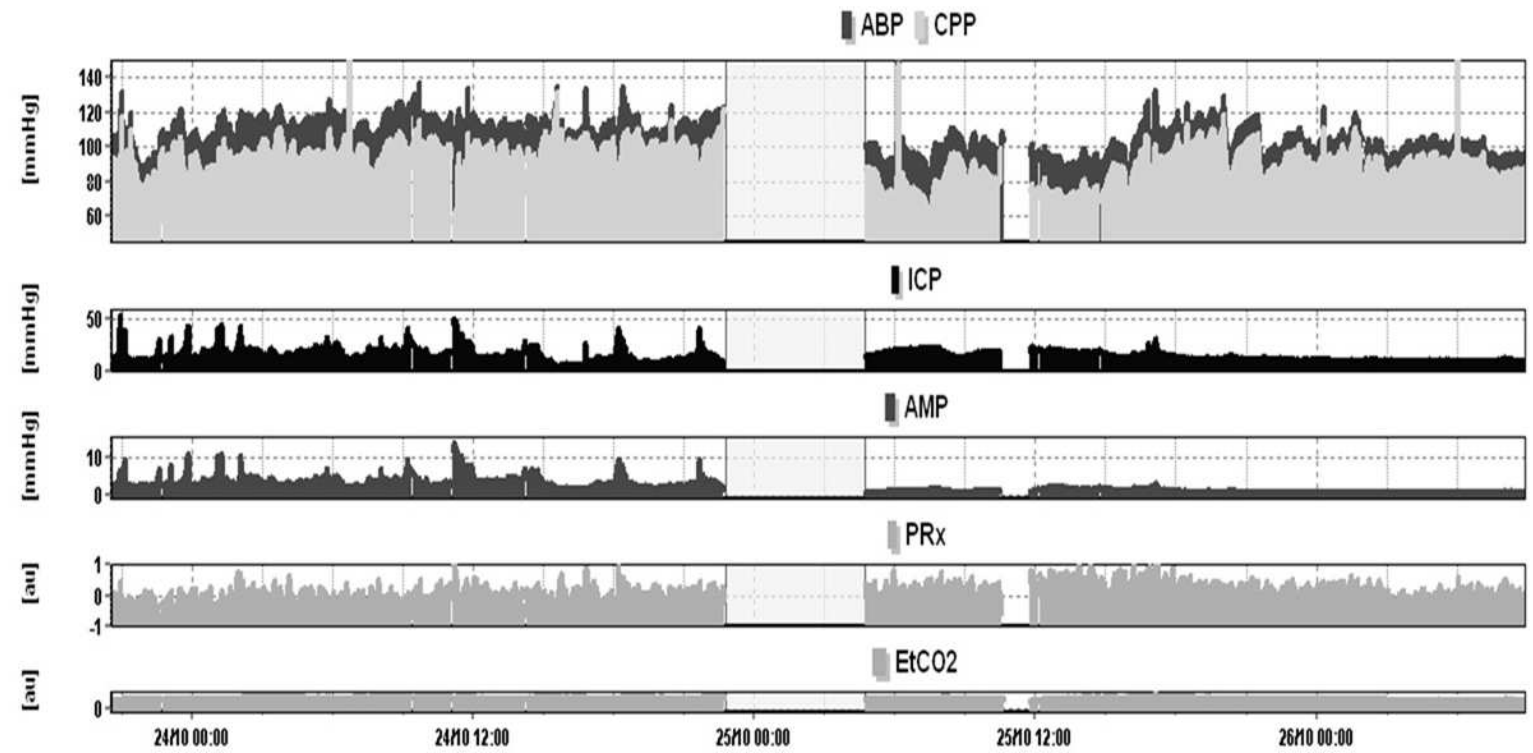

Figure 5: Multimodal brain monitoring changes before and after decompressive craniectomy represented by mean arterial blood pressure (ABP), cerebral perfusion pressure (CPP), intracranial pressure (ICP), amplitude of ICP (AMP), cerebrovascular pressure reactivity (PRx) and endtidal carbon dioxide (ETCO2). 


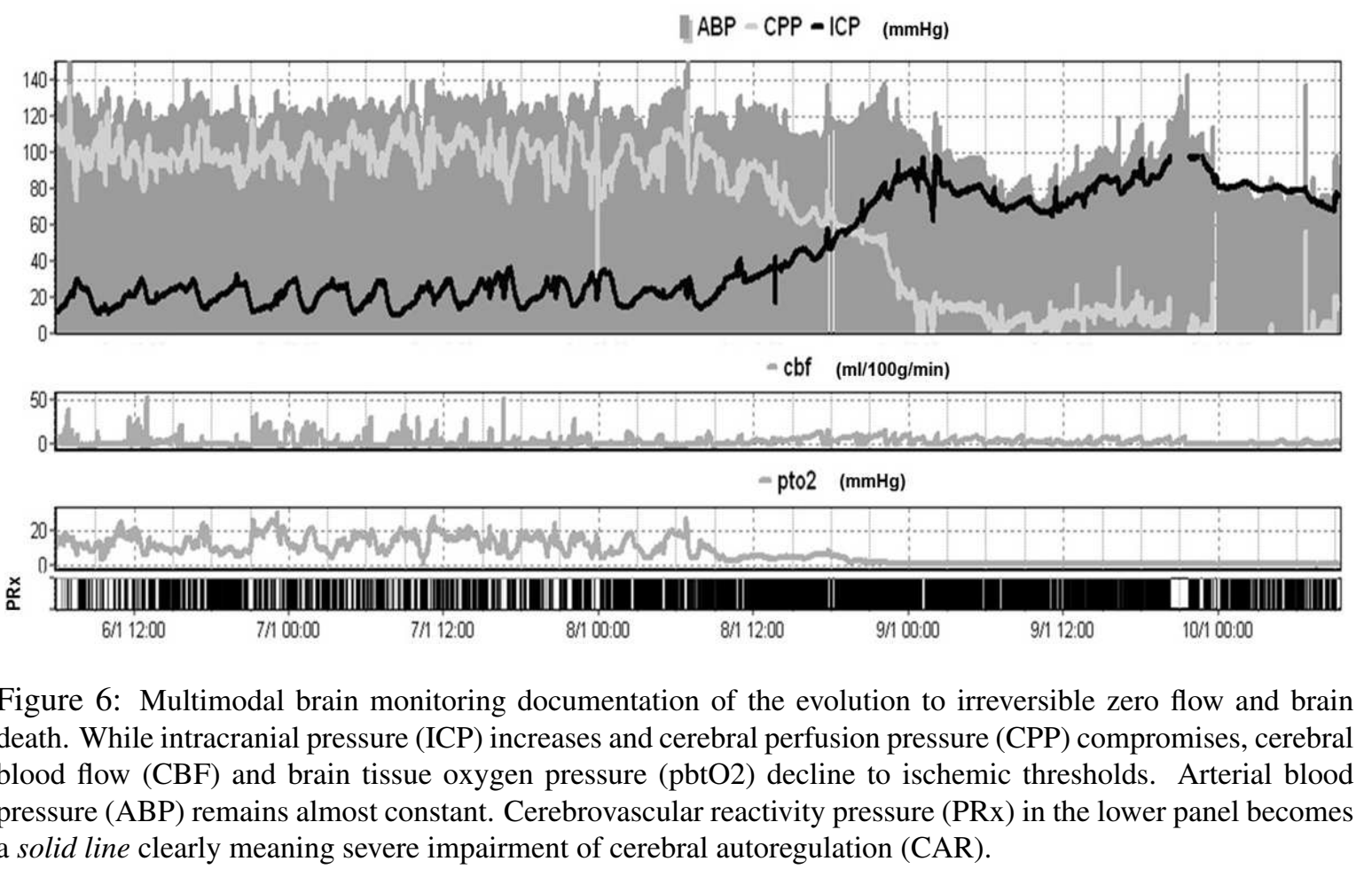

The clinical examples presented in this section regard five adult patients with severe traumatic brain injury. All patients, as already mentioned, developed intracranial hypertension. Patient P1 is a noteworthy example of intracranial hypertension with plateau waves due to the huge number of occurrences, along with a good response to osmotherapy with hypertonic saline medical treatment thus avoiding DC. Four patients, hereafter designated by P2 to P5 were submitted to DC. Two of these, P2 and P3 survived with good outcome while the other two, P4 and P5, evolved to BD.

The results of analysing a total of 2123 segments across all available data from the five patients are summarized in Table 1 . The low signal quality, with many corrupted excerpts, typically found in intensive care recordings, is the cause of the reduced number of segments per day. Firstly, note that not only the percentage of segments fitted with ARFIMA models is higher but also the median length of those segments is larger. Moreover, the order of the ARFIMA models is relevantly lower, with only approximately half of the segments needing an order higher than $p=30$. These findings indicate that the ARFIMA model inherent detrending is superior to the current advanced HRV detrending techniques using smooth priors, leading to a better adjustment to each patient. Thus ARFIMA-GARCH modeling is advantageous to describe HRV dynamics.

To analyse the effect of PW in the dynamics of HRV consider the occurrence of four plateau waves with minimum duration of approximately 9 minutes (events) for patient P1. For each event two segments are considered: the period of time with ICP $>40 \mathrm{mmHg}$, designated by during $P W$ and 1024 beats after ICP normalized below $20 \mathrm{mmHg}$, designated by after $P W$. The results are summarized in Table 2. In segment during $P W, d>0.54$ and the GARCH parameters $u, v$ satisfy $u+v>0.9$, while in segment after $P W, d$ decreases to values below 0.33 in the first three events and $d=0.47$ in the 4 th event. The volatility, measured by $u+v$ also decreases to low values, $u+v<0.65$, in the first three events, with two of them presenting absence of volatility, $u+v=0$. The 4 th event, presenting the higher values for long memory $d=0.47$ and volatility $u+v=0.98$, has the particularity of corresponding to a spontaneous reversion of ICP without osmotherapy. These findings suggest the hypothesis that hypertonic saline not only decreases ICP but also affects the dynamics of HRV in terms of conditional mean and conditional variance. In 


\begin{tabular}{|c|c|c|c|c|c|c|c|}
\hline & \multicolumn{5}{|c|}{ Patients } & \multirow[b]{2}{*}{ Overall } \\
\hline & & P1 & P2 & P3 & P4 & P5 & \\
\hline \multicolumn{2}{|c|}{ Monitorization (approximate length) } & 21 days & 7 days & 16 days & 12 days & 10 days & 66 days \\
\hline \multicolumn{2}{|c|}{ Total number of segments } & 672 & 247 & 405 & 265 & 534 & 2123 \\
\hline \multirow{2}{*}{$\%$ segments with valid models } & AR & $60 \%$ & $53 \%$ & $56 \%$ & $48 \%$ & $49 \%$ & $54 \%$ \\
\hline & ARFIMA & $86 \%$ & $95 \%$ & $88 \%$ & $84 \%$ & $87 \%$ & $87 \%$ \\
\hline \multirow{2}{*}{ Median length (beats) } & $\mathrm{AR}$ & 2162.5 & 1893 & 1776.5 & 1679 & 2870 & 2016 \\
\hline & ARFIMA & 2926.5 & 3047 & 2672 & 2424.5 & 4096 & 3031 \\
\hline \multirow{2}{*}{ median order } & $\mathrm{AR}$ & 36 & 36 & 35 & 36 & 36 & \\
\hline & ARFIMA & 29 & 27 & 31 & 31 & 30 & \\
\hline \multirow{2}{*}{$\begin{array}{c}\% \text { models with } \\
\text { maximum order chosen above } 30\end{array}$} & $\mathrm{AR}$ & $90 \%$ & $86 \%$ & $82 \%$ & $88 \%$ & $81 \%$ & $85 \%$ \\
\hline & ARFIMA & $46 \%$ & $40 \%$ & $51 \%$ & $51 \%$ & $47 \%$ & $47 \%$ \\
\hline
\end{tabular}

Table 1: Features resulting from the modeling of RR series segments, during the whole period of monitoring, of patients P1 to P5 with AR models and ARFIMA-GARCH models.

\begin{tabular}{|c|c|c|c|c|c|c|}
\hline \multirow[b]{2}{*}{ Event } & \multicolumn{3}{|c|}{ during $P W$} & \multicolumn{3}{|c|}{ after $P W$} \\
\hline & $d$ & $u$ & $v$ & $d$ & $u$ & $v$ \\
\hline 1 & 0.55 & 0.07 & 0.9 & 0.33 & 0 & 0 \\
\hline 2 & 0.54 & 0.45 & 0.54 & 0.23 & 0.20 & 0.44 \\
\hline 3 & 0.78 & 0.11 & 0.82 & 0.30 & 0 & 0 \\
\hline 4 & 0.82 & 0.07 & 0.09 & 0.47 & 0.03 & 0.95 \\
\hline
\end{tabular}

Table 2: ARFIMA-GARCH model parameters estimates in RR segments during $P W$ and after $P W$ plateau waves with minimum duration of approximately 9 minutes (events).

fact, Dias and co-authors [11] have shown that the first effect of hypertonic saline administration is an increase of cerebral blood flow with reduction of cerebral vascular resistance while ABP is minimally affected. These effects, although small, may nevertheless be sufficient to induce variations in HRV dynamics.

As mentioned above, the management of the refractory intracranial hypertension of patients P2 to P5 involved a surgical approach by DC. The immediate effect of the surgical procedure in the HRV dynamics was studied by comparing the parametric HRV measures over the admissible models in the periods of 24h before (PRE) and after (POST) DC.

Figure 7 summarizes the distributions of TP, B, LFn and HFn for each patient in PRE and POST decompressive cranietomy, calculated over the segments described in Table 3 In the upper panels, the represented values were obtained from segments for which adequate AR (left boxplots) and ARFIMA-GARCH (right boxplots) models were found. Differences both in median values and dispersion are observable, in particular for LFn and B, both depending on LF. These findings indicate that the detrending strategies (smooth prior detrending and long-memory filtering) have impact in the spectral measures.

Note that the percentage of segments fitted with ARFIMA models is much higher and also the median length of those segments is larger than those fitted with AR models. This fact prompts the 


\begin{tabular}{|c|c|c|c|c|c|c|c|c|c|}
\hline & & \multicolumn{8}{|c|}{ Patients } \\
\hline & & \multicolumn{2}{|c|}{$\mathrm{P} 2$} & \multicolumn{2}{|c|}{ P3 } & \multicolumn{2}{|c|}{ P4 } & \multicolumn{2}{|c|}{ P5 } \\
\hline & & PRE & POS & PRE & POS & PRE & POS & PRE & POS \\
\hline \multicolumn{2}{|c|}{ Total number of segments } & 10 & 30 & 18 & 28 & 21 & 15 & 20 & 27 \\
\hline \multirow{2}{*}{$\%$ segments with valid models } & AR & $50 \%$ & $77 \%$ & $44 \%$ & $37 \%$ & $81 \%$ & $33 \%$ & $65 \%$ & $15 \%$ \\
\hline & ARFIMA & $100 \%$ & $100 \%$ & $100 \%$ & $86 \%$ & $100 \%$ & $100 \%$ & $95 \%$ & $96 \%$ \\
\hline \multirow{2}{*}{ Median length (beats) } & $\mathrm{AR}$ & 1877 & 1653 & 2456.5 & 1553 & 1857 & 1634 & 2680 & 4096 \\
\hline & ARFIMA & 3043.5 & 2277 & 3896 & 2994.5 & 2215 & 3000 & 3358 & 4096 \\
\hline
\end{tabular}

Table 3: Features resulting from modeling RR series segments of patients $\mathrm{P} 2$ to P5, in the periods of 24h before (PRE) and after (POS) DC, with AR and ARFIMA-GARCH models.

study of the spectral measures obtained for all segments for which ARFIMA model was estimated, which are represented in the lower panels of figure 7 Globally, TP decreases after DC while B stays at a comparable level. Regarding the two traditional bands, HFn decreases and LFn increases except in P2. As a matter of fact, the absolute measures HF and LF decrease for all patients except LF in P4. The less marked reduction in LF may be explained by the sustained need of hemodynamic support managed with noradrenaline infusion according to NCCU protocol [11].

The changes of these spectral measures may be related to the decrease of intracranial pressure expected after DC [12]. In spite of absence of relevant differences in the median of B with DC, the dispersion (evaluated by interquartile range) increased. In a recent study by Dias et al [12] over 9 patients submitted to DC (including the four patients P2-P5) the correlation between nonparametric based B and ICP was studied. A positive significant correlation of 0.4 was found in the $12 \mathrm{~h}$ immediately preceding DC, becoming negligible and non significant in the post-DC period.

For patients $\mathrm{P} 4$ and $\mathrm{P} 5$ who evolved to BD, the time of ZF, was documented c.f. Figure 6 It is then possible to study retrospectively HRV dynamics in $24 \mathrm{~h}$ periods from the monitoring restore time after DC to ZF and after ZF. In addition to the expected reduced HRV, ARFIMA-GARCH modeling indicates also less heteroscedasticity in $\mathrm{BD}$, measured by the percentage of segments in each 24h with $u+v \geq 0.65$, Figure 8 . Note that there are the two values for patient P5 in day 6 resulting from the fact that $Z F$ occurred within that $24 \mathrm{~h}$ period. The marked reduction between the two values reflects an immediate effect of ZF. Thus, ARFIMA-GARCH models allow to notice that heteroscedasticity decreases simultaneously with the irreversible compromising of cerebral perfusion pressure.

In summary, ARFIMA-GARCH modeling allows the quantification of long range correlations, $d$, and volatility, $u$ and $v$, which appear to reflect changes in HRV dynamics related both with the acute brain lesion and the medical treatments effects.

\section{Final Comments}

The last decade has witnessed an increase of interest in the characterization of complexity and nonlinearity in HRV with the aim of risk assessment. Here two specific complex/nonlinear characteristics of HRV, long memory and time-varying variance, are considered from a model based parametric point of view. Concerning long memory, several studies in the literature [23, 26], and references there included, indicate that the application of heuristic approaches such as DFA, lead to the same information on HRV. However, the model based ARFIMA approach has the advantage of allowing the design of individualized filters and simultaneously the obtention of clinically interpretable measures such as low and high frequency components in HRV. 
AR versus ARFIMA models (same segments)
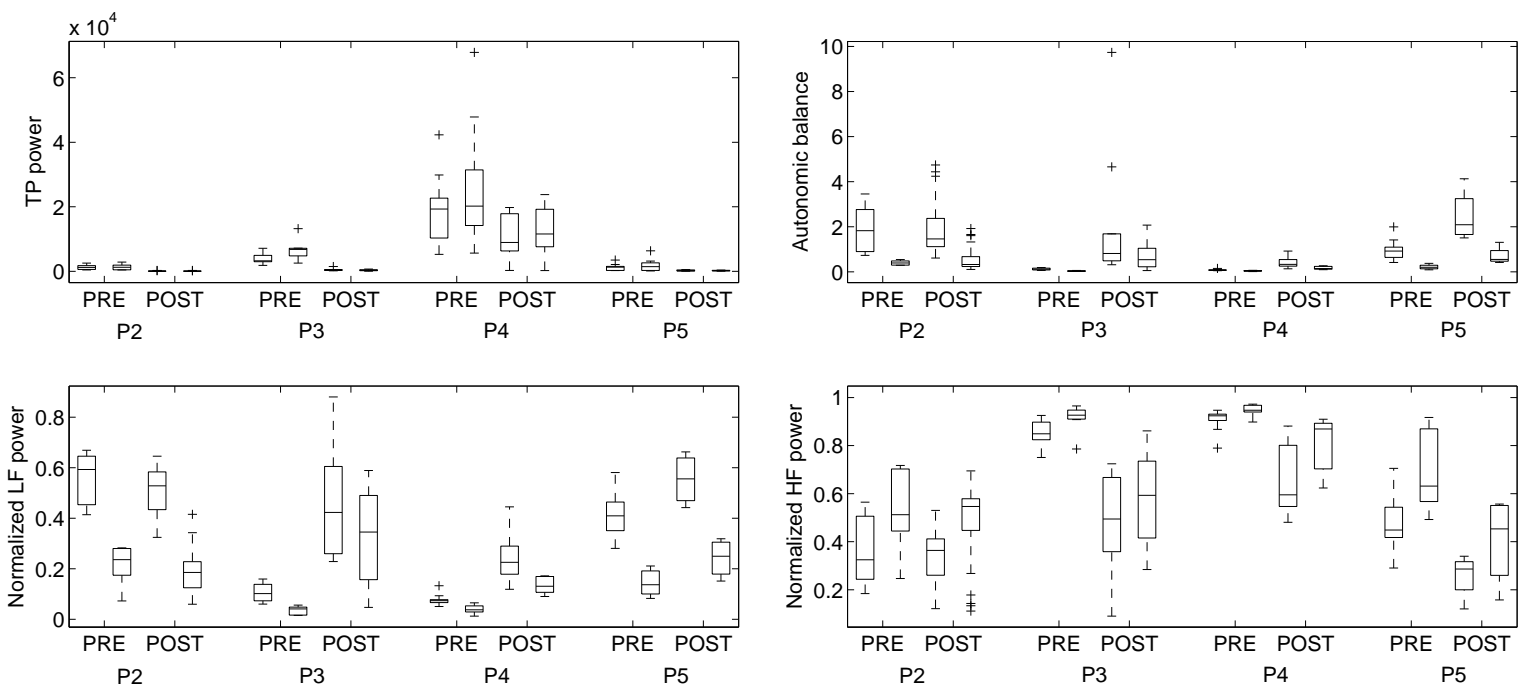

All ARFIMA models
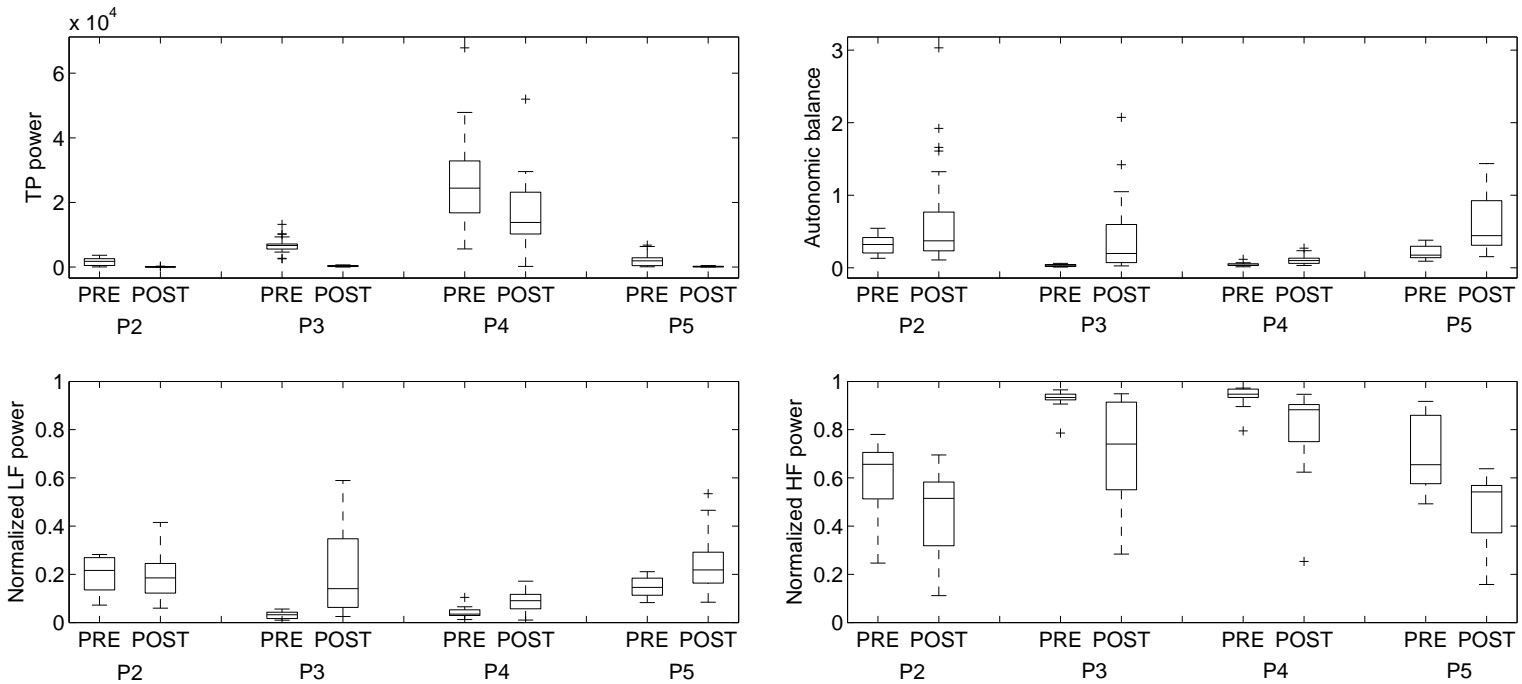

Figure 7: Box plot of spectral measures over TP, B, LFn and HFn for each patient in pre-DC and post-DC: in the upper panel first box plot for each case correspond to AR models and the second to ARFIMA models.

The potential of HRV analysis as a tool to assess the sympathetic and parasympathetic dysfunction of the ANS after ABI has not been fully investigated yet, although Norris et al [37] suggested that HRV could be used to predict the outcome in critically injured patients as one of a set of new vital signs. The parametric ARFIMA-GARCH approach advocated in this study provides new measures to describe the individual patient HRV dynamics, which may be particularly relevant to clinicians who manage critically ill patients with complex medical conditions that change rapidly and sometimes unpredictably. Further studies are warranted to fully appraise ARFIMA-GARCH modeling of HRV in acute brain injury. 


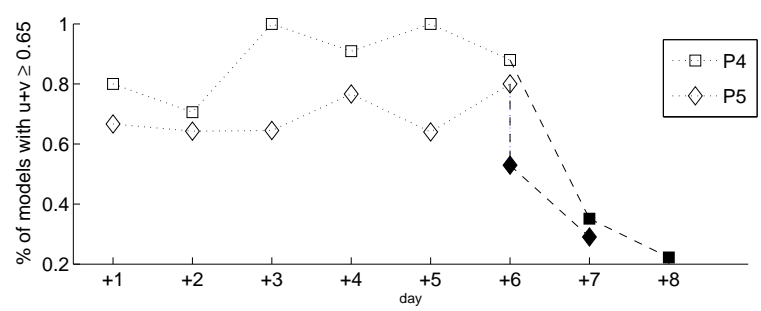

Figure 8: Percentage of models with $u+v \geq 0.65$ for BD patients, P4 and P5. Dark marks correspond to time after zero flow (ZF).

\section{Acknowledgement}

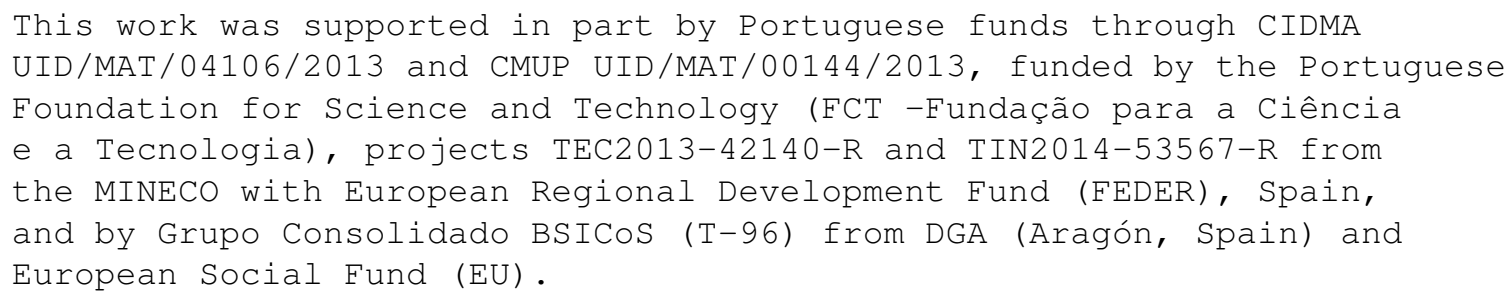

\section{References}

[1] Almeida R, Gouveia S, Rocha AP, Pueyo E, Martínez JP, Laguna P (2006) Exploring QT variability dependence from heart rate in coma and brain death on pediatric patients. IEEE Transactions on Biomedical Engineering 53:1317-1329

[2] Almeida R, Silva MJ, Rocha AP (2013) Exploring QT variability dependence from heart rate in coma and brain death on pediatric patients. Proceedings of Computing in Cardiology Conference (CinC), 2013, 61-64

[3] Baillie RT, Cecen AA, Erkal C (2009) Normal heartbeat series are nonchaotic, nonlinear, and multifractal: New evidence from semiparametric and parametric tests. Chaos 19:028503

[4] Beran J, Feng Y, Ghosh S, Kulik R (2012) Statistics for Long-Memory Processes: Probabilistic Properties and Statistical Methods. Springer

[5] Bianchi AM, Mainardi LT, Cerutti S (2000) Time-frequency analysis of biomedical signals. Transactions of the Institute of Measurement and Control 22:215230

[6] Biswas AK, Scott WA, Sommerauer JF, Luckett PM (2000) Heart rate variability after acute traumatic brain injury in children. Crit Care Med 28(12):3907-3912

[7] Bolea J, Almeida R, Laguna P, Sörnmo L, Martínez JP; BioSigBrowser, biosignal processing interface (2009) Final Program and Abstract Book 9th International Conference on Information Technology and Applications in Biomedicine, ITAB 2009, art. no. 5394301.

[8] Bollerslev T (1986) Generalized autoregressive conditional heteroscedasticity. J Econometr 31:307-327

[9] Buchman TG, Stein PK, Goldstein B (2002) Heart rate variability in critical illness and critical care. Curr Opin Crit Care 8(4):311-315

[10] Dias C, Maia I, Cerejo A, Varsos G, Smielewski P, Paiva JA, Czosnyka M (2014) Pressures, flow, and brain oxygenation during plateau waves of intracranial pressure. Neurocrit Care 21(1):124-32 
[11] Dias C, Silva MJ, Pereira E, Monteiro E, Maia I, Barbosa S, Silva S, Honrado T, Cerejo A, Aries MJH, Smielewski P, Paiva JA, Czosnyka M (2015) Optimal Cerebral Perfusion Pressure Management at Bedside: A Single-Center Pilot Study. Neurocrit Care 23(1):92102

[12] Dias C, Almeida R, Vaz Ferreira A, Silva J, Monteiro E, Cerejo A, Rocha AP (2016) Heart Rate Variability and Multimodal Brain Monitoring before and after Decompressive Craniectomy in Traumatic Brain Injury. Intensive Care Medicine Experimental 2016, 4(Suppl 1):30:209

[13] Gang, Y, Malik, M (2002). Heart rate variability in critical care medicine. Current Opinion in Critical Care, 8(5): 3715

[14] Goldberger AL, Amaral LAN, Glass L, Hausdorff JM, Ivanov PC, Mark RG, Mietus JE, Moody GB, Peng CK, Stanley HE (2000) Physiobank, physiotoolkit and physionet: Components of a new research resource for complex physiologic signals. Circulation 101:e $215 \mathrm{e} 220$

[15] Haji-Michael PG, Vincent JL, Degaute JP, van de Borne P (2000) Power spectral analysis of cardiovascular variability in critically ill neurosurgical patients. Crit Care Med. 28(7):25782583

[16] Hosking JRM (1981) Fractional differencing. Biometrika 68:165-176

[17] Hu YM, Tsoukalas C (2006) Conditional volatility properties of sleep-disordered breathing. Computers in Biology and Medicine 36(3):303-312

[18] Hurvich CM, Ray BK (1995) Estimation of the memory parameter for nonstationary or noninvertible fractionally integrated processes. J Time Series Analysis 16:17-41

[19] Kahraman, S, Dutton, RP, Hu, P, Stansbury, L, Xiao, Y, Stein, D M, Scalea, T M (2010). Heart Rate and Pulse Pressure Variability are Associated With Intractable Intracranial Hypertension After Severe Traumatic Brain Injury. Journal of Neurosurgical Anesthesiology, 22(4), 296302

[20] Kakar V, Nagaria J, John Kirkpatrick P (2009) The current status of decompressive craniectomy. Br J Neurosurg 23(2):147-157

[21] Kamath MV, Watanabe M, Upton A (eds) (2013) Heart Rate Variability (HRV) Signal Analysis: Clinical Applications. CRC Press, US

[22] Leite A, Rocha AP, Silva ME, Costa O (2006) Modelling long-term heart rate variability: an ARFIMA approach. Biomedizinische Technik 51:215219

[23] Leite A, Rocha AP, Silva ME, Gouveia S, Carvalho J,Costa, O, (2007) Long-range dependence in heart rate variability data: ARFIMA modelling vs detrended fluctuation analysis. Proceedings of Computers in Cardiology. 34:21-24

[24] Leite A, Rocha AP, Silva ME (2009) Long memory and volatility in HRV: an ARFIMAGARCH approach. Proceedings of Computers in Cardiology. 36:165168

[25] Leite A, Rocha AP, Silva ME (2013) Beyond long memory in heart rate variability: An approach based on fractionally integrated autoregressive moving average time series models with conditional heteroscedasticity. Chaos: An Interdisciplinary Journal of Nonlinear Science. 23(2):023103

[26] Leite A, Silva ME, Rocha AP (2013) Scaling Exponents in Heart Rate Variability. In J. Lita da Silva, F. Caeiro, I. Natrio, and C.A. Braumann, editors, Advances in Regression, Survival Analysis, Extreme Values, Markov Processes and Other Statistical Applications, pages 259-267. Springer, 2013.

[27] Leite A, Rocha AP, Silva ME (2016) Modeling volatility in Heat Rate Variability. 2016 Annual International Conference of the IEEE Engineering in Medicine and Biology Society (EMBC). IEEE Engineering in Medicine and Biology Society Conference Proceedings:3582-3585 
[28] Ljung GM, Box GEP (1978) On a measure of lack of fit in time series models. Biometrika 65: 297-303

[29] Luis A, Santos AS, Dias C, Almeida R, Rocha AP (2015) Heart rate variability during plateau waves of intracranial pressure: A pilot descriptive study. 2015 Annual International Conference of the IEEE Engineering in Medicine and Biology Society (EMBC). IEEE Engineering in Medicine and Biology Society Conference Proceedings:6142-6145

[30] Maddala GS, Kim IM (1999) Unit Roots, Cointegration, and Structural Change (Themes in Modern Econometrics). Cambridge University Press, UK

[31] Mainardi LT, Bianchi AM, Cerutti S (2002) Time-frequency and time-varying analysis for assessing the dynamic responses of cardiovascular control. Critical Reviews in Biomedical Engineering 30:175217

[32] Martínez JP, Almeida R, Olmos S, Rocha AP, Laguna P (2004) Wavelet-based ECG delineator: evaluation on standard databases. IEEE Transactions on Biomedical Engineering 51:570-581

[33] Mazzeo AT, LaMonaca E, DiLeo R, Vita G, Santamaria LB (2011) Heart rate variability: a diagnostic and prognostic tool in anesthesia and intensive care. Acta Anaesthesiologica Scandinavica 55(7): 797-811

[34] Mowery NT, Norris PR, Riordan W, Jenkins JM, Williams AE, Morris JA, Jr (2008) Cardiac uncoupling and heart rate variability are associated with intracranial hypertension and mortality: a study of 145 trauma patients with continuous monitoring. J Trauma 65(3):621627

[35] Nakagawa T, Ashwal S, Mathur M, Mysore M (2012) Guidelines for the determination of brain death in infants and children: an update of the 1987 task force recommendationsexecutive summary. Annals of neurology 71(4):573-585

[36] Neidzwiecki M (2000) Identification of Time-Varying Processes. Wiley

[37] Norris PR, Morris JA, Ozdas A, Groga EL, Williams AE (2005) Heart Rate Variability Predicts Trauma Patient Outcome as Early as $12 \mathrm{~h}$ : Implications for Military and Civilian Triage. Journal of Surgical Research, 129(1):122-128

[38] Rajendra Acharya U, Paul Joseph K, Kannathal N, Lim CM, Suri JS (2006) Heart rate variability: a review. Med Biol Eng Comput. 44(12):1031-1051

[39] Robinson PM (1995). Gaussian semiparametric estimation of long range dependence. The Annals of Statistics. 23(5):1630-1661.

[40] Rocha AP, Almeida R, Leite A, Silva MJ, Silva ME (2014) Long-term HRV in critically ill pediatric patients: comma versus brain death. In: Computing in Cardiology 41:89-92

[41] Rocha AP, Leite A, Gouveia S, Lago P, Costa O, Freitas AF (2000) Spectral characterization of long-term ambulatory heart rate variability signals. 5th IMA Conference on Mathematics in Signal Processing

[42] Rocha AP, Leite A, Silva ME (2016) Volatility Leveraging in Heart Rate: health vs disease. Proceedings of Computing in Cardiology. 43.

[43] Ryan ML, Thorson CM, Otero CA, Vu T, Proctor KG (2011) Clinical applications of heart rate variability in the triage and assessment of traumatically injured patients. Anesthesiology research and practice 2011:416590

[44] Sassi R, Cerutti S, Lombardi F, Malik M, Huikuri H, Peng C-K, Schmidt G, Yamamoto Y (2015) Advances in heart rate vari- ability signal analysis: joint position statement by the eCardiology ESC Working Group and the European Heart Rhythm Association co-endorsed by the Asian Pacific Heart Rhythm Society Europace 17(9):1341-1353

[45] Signorini MG, Sassi R, Cerutti S (2001) Working on the NOLTISALIS Database: Measurement of nonlinear properties in heart rate variability signals. Proceedings of IEEE- EMBS International Conference 2001 547-550 
[46] Stein PK, Bosner MS, Kleiger RE, Conger BM (1994) Heart rate variability: a measure of cardiac autonomic tone. Am Heart J.127(5):1376-1381

[47] Sykora M, Czosnyka M, Liu X, Donnelly J, Nasr N, Diedler J, et al (2016) Autonomic Impairment in Severe Traumatic Brain Injury: A Multimodal Neuromonitoring Study. Crit Care Med 44(6):1173-1181

[48] Tarvainen MP, Ranta-aho, PO, Karjalainen, PA (2002) An advanced detrending method with application to HRV analysis. IEEE Trasactions in Biomedical Engineering 49(2):172 - 175

[49] Task Force of the European Society of Cardiology and the North American Society of Pacing and Electrophysiology (1996) Heart rate variability. Standards of measurement, physiological interpretation, and clinical use. Eur Heart J. 17(3):354-381

[50] Vallbona, C, Cardus, D, Spencer, WA, Hoff, HE (1965). Patterns of sinus arrhythmia in patients with lesions of the central nervous system. The American Journal of Cardiology, 16(3), 37989

[51] Vanderlei LC, Pastre CM, Hoshi RA, Carvalho TD, Godoy MF (2009) Basic notions of heart rate variability and its clinical applicability. Rev Bras Cir Cardiovasc24(2):205-217

[52] Velasco C (1999) Gaussian semiparametric estimation of nonstationary time series. J Time Series Analalysis 20:87127

[53] Wijdicks EFM (2001) The diagnosis of brain death N Engl J Med 344:1215-1221

[54] Wilcox, RR (2010). Fundamentals of Modern Statistical Methods 2ndEd Academic Press.

[55] Winchell RJ, Hoyt DB (1996) Spectral analysis of heart rate variability in the ICU: a measure of autonomic function. J Surg Res 63(1):11-16 